\title{
School Drug Testing: A Critical Review of the Literature
}

\author{
Daniel T.L. Shek $k^{1,2,3,4}$ \\ ${ }^{1}$ Department of Applied Social Sciences, The Hong Kong Polytechnic University; \\ 2Department of Sociology, East China Normal University, Shanghai; ${ }^{3}$ Public Policy \\ Research Institute, The Hong Kong Polytechnic University; ${ }^{4}$ Kiang Wu Nursing \\ College of Macau \\ E-mail: daniel.shek@polyu.edu.hk
}

Received December 28, 2009; Revised January 25, 2010; Accepted January 27, 2010; Published March 5, 2010

This paper explores the question of whether school drug testing is an effective solution to tackle adolescent substance abuse problems. Research studies in major academic databases and Internet websites are reviewed. Several observations are highlighted from the review: (1) there are few research studies in this area, particularly in different Chinese contexts; (2) the quality of the existing studies was generally low; and (3) research findings supporting the effectiveness of school drug testing were mixed. Methodological issues underlying quantitative and qualitative evaluation studies of the effectiveness of school drug testing are also discussed.

KEYWORDS: drug testing, adolescent substance abuse, abuse detection, adolescents

\section{INTRODUCTION}

A survey of the websites of several international organizations (e.g., Office on Drugs and Crime of the United Nations, International Narcotics Control Board, National Institute of Drug Abuse in the United States, and European Monitoring Center for Drugs and Drug Addiction) shows that illicit drug use is a thorny global problem to be resolved. Probably because of the influence of the popular culture and youth subculture, substance abuse among young people has also become an acute global problem[1,2]. With reference to the findings reported in some of the major databases on adolescent development, such as Monitoring the Future, Youth Risk Behavior Surveillance (YRBS), and National Household Survey on Drug Abuse (NHSDA), adolescent substance abuse is a concern for policy makers and health professionals[3]. From the results of the 2008 National Survey on Drug Use and Health, it was found that $9.3 \%$ of youths aged $12-17$ were current illicit drug users[4].

To tackle the gradual worsening of adolescent drug abuse, school drug testing has been adopted in some Western countries in order to cope with the problem. In 1995, the U.S. Supreme Court approved drug testing for student athletes in public high schools. In 2002, the U.S. Supreme Court broadened the ruling to include all students taking part in competitions against students at other schools in after-school activities[5]. Ever since its inception, there has been much debate on the necessity and value of student drug testing, particularly its effectiveness. Roche et al.[6] reviewed the theories, assumptions, and limitations of the underlying rationales for school drug testing. They also reviewed some of the studies in 
the field and concluded that the quality of the studies was generally low. Although the study of Roche et al.[6] is a pioneering attempt to review some of the studies in the field, there are three limitations. First, the studies under review were not exhaustive, as some of the studies reported in academic journals and the Internet were not included. Second, although the quality of the studies under review was discussed in the paper, the details (e.g., problems of the design, methodology and data analyses, biased conclusions, etc.) were not included. Third, findings that support the effectiveness of school drug testing and those that oppose it were not separately reported. Against this background, the present study attempted to review the literature on the effectiveness of school drug testing. Findings from the literature that support it and those that criticize it are individually presented. Besides, quality of the studies is evaluated in detail. Finally, methodological issues intrinsic to quantitative and qualitative studies of the effectiveness of school drug testing are also discussed.

\section{METHODS}

\section{Search Strategy}

The primary aim of this systematic review was to explore the effectiveness of the contentious issue, school drug testing. Searches were undertaken within the major academic databases: PsycINFO, Social Work Abstracts, Medline, CINAHL, and Sociological Abstracts, using multiple keywords: random drug test or drug testing or school drug testing or drug detection. In addition, empirical studies reported in the websites on the Internet were also reviewed using the above terms. The studies under review in the current study are outlined in Appendix 1. Some of the authoritative websites on school drug testing are presented in Table 1.

TABLE 1

Internet Websites on School Drug Testing

\begin{tabular}{|c|c|}
\hline Resource & Website \\
\hline $\begin{array}{l}\text { American Civil Liberties Union - A Test You } \\
\text { Can't Study For: Special Web Feature on } \\
\text { Student Drug Testing }\end{array}$ & http://www.aclu.org/drugpolicy/testing/10845res20021021.html \\
\hline Drug Policy Alliance Network & http://www.drugpolicy.org/law/drugtesting/students/ \\
\hline Monitoring the Future & http://www.monitoringthefuture.org \\
\hline $\begin{array}{l}\text { Office of Safe and Drug-Free Schools } \\
\text { (OSDFS), U.S. Department of Education }\end{array}$ & http://www.ed.gov/about/offices/list/osdfs/index.html \\
\hline $\begin{array}{l}\text { Prevention Resources and Information on Drug } \\
\text { Education (PRIDE) }\end{array}$ & http://www.prideprevention.org/ \\
\hline Student Drug Testing Coalition & http://www.studentdrugtesting.org/ \\
\hline Students for Sensible Drug Policy (SSDP) & http://www.DAREgeneration.com \\
\hline The Association for Addiction Professionals & http://www.naadac.org/ \\
\hline
\end{tabular}

\section{RESULTS}

Several observations can be highlighted from the review. First, not many studies have been conducted to examine the effectiveness of school drug testing since its introduction. With particular reference to the Chinese culture, no study has been conducted in different Chinese contexts. Second, most of the empirical studies were cross-sectional in nature (e.g., surveys and qualitative interviews) and not many 
experimental studies have been conducted. Third, while there are findings from studies that are in support of school drug testing (Table 2), there are also some that do not (Table 3). Fourth, as shown in Table 4, quality of the existing studies was generally not high; therefore, doubt is cast on their conclusions on the effectiveness of drug test. Added to this, there were few well-designed quantitative studies and wellcrafted qualitative evaluation studies in the field.

\section{DISCUSSION}

Despite the heightened public concern for school drug testing and its controversial nature, empirical studies that examine the effectiveness of drug testing in the school context are, surprisingly, limited in number. From the perspective of evidence-based practice, research studies play an indispensable role in clarifying the effectiveness of school drug testing and providing support for the policy. As Chinese people constitute roughly one-fifth of the world's population, the absence of school drug testing research is definitely undesirable, particularly in view of the fact that mandatory drug testing is legally acceptable in mainland China. Furthermore, research on school drug testing is indispensable when voluntary school drug testing was implemented in the Tai Po district of Hong Kong on a trial basis in 2009/10 school year.

The present review shows that there are mixed findings on the effectiveness of school drug testing. It is noteworthy that while there are findings that indicate that drug testing had no positive effect, there are findings that support the effectiveness of school drug testing. This picture is also clearly shown in the study of Goldberg et al.[7], which is one of the few prospective trials in the field. As pointed out by Goldberg et al.[7], "although these findings may differ in other schools or regions of the United States, this study lends credence to some DAT deterrent effects, especially for past year use for drugs, at two time points, and for drugs and alcohol at two time points. However, because some substance abuse mediators appeared to worsen and past month substance use never changed, more research should be performed to assess the policy of drug and alcohol testing's overall effects" (p. 428, italics added). Similarly, Knight and Levy[8], in an editorial of the Journal of Adolescent Health[8], pointed out that "although we might hope that the present study by Goldberg would help to end the national debate, this hope is unlikely to be realized on the basis of this report, which includes ample 'evidence' to fuel the fire on both sides" ( $p$. 419).

As far as the quality of the studies under review is concerned, the review shows that the quality of the existing studies was not high. In addition, it is noteworthy that the findings in the studies under review cannot give a definitive answer to the question of whether school drug testing is effective. For example, although a large sample size was used in the study of Yamaguchi et al.[9], the major limitation was its cross-sectional design because "one cannot make definitive causal interpretations regarding effects of drug testing; only a panel design in a randomized or natural experiment can do so. Perhaps schools that instituted drug testing initially had higher use, and drug testing reduced those levels to levels similar to those at other schools" (p. 164). With the aim to explore the association between student drug use and drug-testing policies in schools, Yamaguchi et al.[9] concluded that "while lack of evidence for the effectiveness of drug testing is not definitive, results suggest that drug testing in schools may not provide a panacea for reducing student drug use that some (including some on the Supreme Court) had hoped" (p. 164). However, probably because of the large sample involved, this study has commonly been taken as evidence against school drug testing.

Obviously, the sustainability of school drug testing depends principally on the amount and quality of research evidence supporting its value and effectiveness. There are two lines of evaluation research that should be done in the future. To begin with, quantitative research utilizing experimental designs should be conducted. However, there are at least six issues that should be addressed in experimental studies. First, selection bias (pregroup differences) may confound the results. Studies utilizing pre-experimental designs, such as the one conducted by Yamaguchi et al.[9], are particularly vulnerable to this threat. Second, it is noteworthy that a drug testing scheme will heighten other schools' sensitivity to drug prevention, which may minimize the treatment effect in the experimental groups. Third, it is possible that 
TABLE 2

A Summary of Findings that Support School Drug Testing

\begin{tabular}{|c|c|c|c|c|c|c|}
\hline Study & Study Design & Setting & Sample & Intervention & Outcomes assessed & Findings \\
\hline $\begin{array}{l}\text { Coombs and Ryan } \\
(1990)\end{array}$ & $\begin{array}{l}\text { Cross-sectional survey } \\
\text { and in-depth } \\
\text { interview }\end{array}$ & $\begin{array}{l}21 \text { intercollegiate teams, } \\
\text { USA }\end{array}$ & $\begin{array}{l}624 \text { intercollegiate } \\
\text { athletes }\end{array}$ & $\begin{array}{l}\text { Mandatory drug } \\
\text { testing } \\
\text { program }\end{array}$ & $\begin{array}{ll}\text { - Identification of student } \\
\text { drug users } \\
\text { - Prevention of continued } \\
\text { drug use }\end{array}$ & $\begin{array}{l}\text { Drug testing proved to be } \\
\text { effective: } \\
\text { - Identification of drug users } \\
\text { - Prevention of continued drug } \\
\text { use } \\
\text { - Reduction of drug use found } \\
\text { in most of drug-using athletes }\end{array}$ \\
\hline $\begin{array}{l}\text { Coombs and Coombs } \\
\text { (1991) }\end{array}$ & $\begin{array}{l}\text { Cross-sectional survey } \\
\text { and in-depth } \\
\text { interview }\end{array}$ & $\begin{array}{l}21 \text { intercollegiate teams, } \\
\text { USA }\end{array}$ & $\begin{array}{l}500 \text { intercollegiate } \\
\text { athletes }\end{array}$ & $\begin{array}{l}\text { Mandatory drug } \\
\text { testing } \\
\text { program }\end{array}$ & $\begin{array}{l}\text { Assessment of students' } \\
\text { morale and psychological } \\
\text { well-being }\end{array}$ & $\begin{array}{l}\text { Most students were not affected } \\
\text { by drug testing. } \\
\text { Positive benefits: } \\
\text { - Promote awareness of } \\
\text { negative drug effects } \\
\text { - Offer socially acceptable way } \\
\text { to resist drug use } \\
\text { Enhance athletic and } \\
\quad \text { academic performance }\end{array}$ \\
\hline $\begin{array}{l}\text { DuPont, Campbell, } \\
\text { and Mazza (2002) }\end{array}$ & Cross-sectional survey & Nine high schools, USA & $\begin{array}{l}\text { School administrators, } \\
\text { counselors, athletic } \\
\text { directors, drug- } \\
\text { prevention } \\
\text { coordinators }\end{array}$ & $\begin{array}{l}\text { Student drug } \\
\text { testing }\end{array}$ & $\begin{array}{ll}\text { - } & \text { Students' reported drug } \\
\text { - } & \text { use } \\
\text { Disciplinary problems }\end{array}$ & $\begin{array}{l}\text { Decreased students' illicit drug } \\
\text { use } \\
\text { Reduced disciplinary problems: } \\
\text { - Lowered detention rate for } \\
\quad \text { disruptive behavior } \\
\text { - } \quad \text { Reduced student arrests }\end{array}$ \\
\hline McKinney (2002) & Cross-sectional survey & $\begin{array}{l}83 \text { high schools, } \\
\text { Indiana, USA }\end{array}$ & $\begin{array}{l}83 \text { high school } \\
\text { principals }\end{array}$ & $\begin{array}{l}\text { Mandatory } \\
\text { random drug } \\
\text { testing in } \\
1999-2000\end{array}$ & $\begin{array}{l}\text { Compare two academic } \\
\text { years: } 1999-2000 \text { (when } \\
\text { drug testing policies were } \\
\text { in effect) to 2000-2001 } \\
\text { (when random drug } \\
\text { testing policies were } \\
\text { suspended): } \\
\text { - Students' reported illicit } \\
\text { drug use } \\
\text { - Students' alcohol use } \\
\text { - Students' suspension } \\
\text { or expulsion due to } \\
\text { drug or alcohol use }\end{array}$ & $\begin{array}{l}\text { After the suspension of random } \\
\quad \text { drug testing program: } \\
\text { - Increase in illicit drug use } \\
\text { - Increase in alcohol use } \\
\text { - Statistically significant } \\
\text { increase in students' } \\
\text { suspension or expulsion }\end{array}$ \\
\hline McKinney (2003) & $\begin{array}{l}\text { Cross-sectional survey } \\
\text { Mckinney's (2002) } \\
\quad \text { follow-up }\end{array}$ & $\begin{array}{l}59 \text { high schools, } \\
\text { Indiana, USA }\end{array}$ & $\begin{array}{l}59 \text { high school } \\
\text { principals }\end{array}$ & $\begin{array}{l}\text { Mandatory, } \\
\text { random drug } \\
\text { testing } \\
\text { program }\end{array}$ & $\begin{array}{l}\text { Students' reported drug and } \\
\text { alcohol use when drug } \\
\text { testing reimplemented }\end{array}$ & $\begin{array}{l}\text { Drug testing discouraged } \\
\text { students' drug and alcohol } \\
\text { use } \\
\text { Decreased students' drug } \\
\text { and alcohol use }\end{array}$ \\
\hline McKinney (2005) & $\begin{array}{l}\text { Cross-sectional survey } \\
\text { McKinney's (2003) } \\
\text { follow-up }\end{array}$ & $\begin{array}{l}56 \text { high schools, } \\
\text { Indiana, USA }\end{array}$ & $\begin{array}{l}56 \text { high school } \\
\text { principals }\end{array}$ & $\begin{array}{l}\text { Mandatory, } \\
\text { random, } \\
\text { suspicion-less } \\
\text { drug testing } \\
\text { program }\end{array}$ & $\begin{array}{ll}\text { - } & \text { Students' reported illicit } \\
\text { drug use } \\
\text { - } & \text { Students' participation } \\
\text { in athletic program } \\
\text { - Students' academic } \\
\text { performance }\end{array}$ & $\begin{array}{l}\text { - Reduction in students' drug } \\
\text { use } \\
\text { - Rise in student participation } \\
\text { in athletic program } \\
\text { Schools with drug testing: } \\
\text { - } \quad \text { Above average in state } \\
\text { graduation test } \\
\text { - Graduation rate higher than } \\
\text { state average }\end{array}$ \\
\hline Mason (2003) & Cross-sectional survey & High schools, USA & $\begin{array}{l}620 \text { high school } \\
\text { students }\end{array}$ & $\begin{array}{c}\text { Drug testing } \\
\text { program }\end{array}$ & $\begin{array}{l}\text { - Students' attitude } \\
\text { toward drug testing }\end{array}$ & $\begin{array}{l}\text { - Neutral attitude on drug } \\
\text { - } \quad \text { Mosting in most students } \\
\text { in younger students } \\
\text { - } \quad \text { Drug testing less accepting } \\
\text { in students with stronger } \\
\text { prodrug attitude }\end{array}$ \\
\hline $\begin{array}{l}\text { Goldberg, Elliot, } \\
\text { MacKinnon, Moe, } \\
\text { Kuehl, Nohre, and } \\
\text { Lockwood (2003) }\end{array}$ & $\begin{array}{l}\text { Longitudinal survey } \\
\quad 1999-2000\end{array}$ & $\begin{array}{l}\text { Two high schools, } \\
\text { Oregon, USA }\end{array}$ & $\begin{array}{l}\text { Athletes vs. } \\
\text { nonathletes: } \\
\text { T1: } 276 \text { vs. } 507 \\
\text { T2: } 159 \text { vs. } 338\end{array}$ & $\begin{array}{l}\text { Mandatory drug } \\
\text { testing } \\
\text { program for } \\
\text { student } \\
\text { athletes }\end{array}$ & $\begin{array}{l}\text { - Students' past 30-day } \\
\text { drug use } \\
\text { - } \quad \text { Students' attitude and } \\
\text { beliefs on drug testing }\end{array}$ & $\begin{array}{l}\text { Reduction in the number of } \\
\text { student athletes to use drug } \\
\text { in the past } 30 \text { days } \\
\text { - Positive attitude toward drug } \\
\text { testing in intervention and } \\
\text { control groups }\end{array}$ \\
\hline $\begin{array}{l}\text { Evans, Reader, Liss, } \\
\text { Wiens, and Roy } \\
(2006)\end{array}$ & $\begin{array}{l}\text { Cross-sectional survey } \\
\text { conducted before } \\
\text { drug testing } \\
\text { implementation }\end{array}$ & $\begin{array}{l}\text { Two rural high schools, } \\
\text { North Florida, USA }\end{array}$ & $\begin{array}{l}1,011 \text { students from } 9^{\text {th }} \\
\text { to } 11^{\text {th }} \text { grade }\end{array}$ & $\begin{array}{l}\text { Random } \\
\text { suspicion-less } \\
\text { drug testing } \\
\text { program }\end{array}$ & $\begin{array}{l}\text { - Students' perceived } \\
\text { fairness of drug testing } \\
\text { policy } \\
\text { - Students' predicted } \\
\text { effectiveness to reduce } \\
\text { drug use }\end{array}$ & $\begin{array}{l}\text { - Perceived drug problem } \\
\text { found as robust predictor of } \\
\text { perceived policy fairness. } \\
\text { - Most students perceived that } \\
\text { drug testing would be } \\
\text { effective to reduce drug use. }\end{array}$ \\
\hline $\begin{array}{l}\text { Evans-Whipp, Bond, } \\
\text { Toumbourou, and } \\
\text { Catalano (2007) }\end{array}$ & Cross-sectional survey & $\begin{array}{l}\text { International Youth } \\
\text { Development Study } \\
\text { data 2003: } \\
\text { - } 104 \text { schools, } \\
\text { Washington, USA } \\
\text { 101 schools, } \\
\text { Victoria, Australia }\end{array}$ & $\begin{array}{l}\text { Washington: } \\
1,934 \text { students } \\
1,886 \text { parents } \\
\text { Victoria: } \\
1,942 \text { students } \\
1,858 \text { parents }\end{array}$ & $\begin{array}{c}\text { Drug testing } \\
\text { program }\end{array}$ & $\begin{array}{ll}\text { - } & \text { Students' reported drug } \\
\text { use } \\
\text { - } & \text { Parents' and students' } \\
& \text { awareness of policy }\end{array}$ & $\begin{array}{l}\text { - Drug testing policy } \\
\text { associated with decreased } \\
\text { student drug use } \\
\text { - The message of harm } \\
\text { reduction associated with } \\
\text { reduced drug use } \\
\text { - Parents and students aware } \\
\text { of school policy orientation }\end{array}$ \\
\hline $\begin{array}{l}\text { Goldberg, Elliot, } \\
\text { MacKinnon, Moe, } \\
\text { Kuehl, Yoon, } \\
\text { Taylor, and } \\
\text { Williams (2007) }\end{array}$ & $\begin{array}{l}\text { 2-year prospective } \\
\text { randomized } \\
\text { controlled study of a } \\
\text { single cohort }\end{array}$ & $\begin{array}{l}14 \text { school districts, } \\
\text { Oregon, USA }\end{array}$ & $\begin{array}{l}\text { - } 653 \text { students in } \\
\text { five high schools } \\
\text { with drug testing } \\
\text { - } 743 \text { students in } \\
\text { six control } \\
\text { schools }\end{array}$ & $\begin{array}{l}\text { Random drug and } \\
\text { alcohol testing } \\
\text { in high school } \\
\text { athletes }\end{array}$ & $\begin{array}{ll}\text { - } & \text { Students' past-year } \\
\text { reported drug use } \\
\text { - } & \text { Students' past-month } \\
\text { reported drug use }\end{array}$ & $\begin{array}{l}\text { Reduced students' past-year drug } \\
\text { use in two of four follow-ups }\end{array}$ \\
\hline Barrington (2008) & $\begin{array}{l}\text { Quasi-experimental } \\
\text { mixed-methods } \\
\text { sequential } \\
\text { explanatory design }\end{array}$ & $\begin{array}{l}\text { Two rural, low-income } \\
\text { public secondary } \\
\text { school districts, USA }\end{array}$ & $\begin{array}{l}\text { 1,048 high school } \\
\text { students from } 6^{\text {th }} \text { to } \\
12^{\text {th }} \text { grade, and four } \\
\text { school } \\
\text { administrators }\end{array}$ & $\begin{array}{l}\text { Voluntary, } \\
\text { randomized, } \\
\text { student drug } \\
\text { testing } \\
\text { program }\end{array}$ & Drug testing efficacy & $\begin{array}{l}\text { Qualitative findings: } \\
\text { - Students with intensive drug } \\
\text { abuse service needs } \\
\text { identified } \\
\text { - Enhance school bonding and } \\
\text { connectedness }\end{array}$ \\
\hline $\begin{array}{l}\text { Ringwalt, Vincus, } \\
\text { Ennett, Hanley, } \\
\text { Bowling, } \\
\text { Yacoubian, and } \\
\text { Rohrbach (2009) }\end{array}$ & $\begin{array}{l}\text { Cross-sectional survey } \\
\text { in spring } 2005\end{array}$ & $\begin{array}{l}\text { School districts from a } \\
\text { national random } \\
\text { sample, USA }\end{array}$ & $\begin{array}{l}\text { 1,612 drug prevention } \\
\text { coordinators from } \\
1,922 \text { school } \\
\text { districts }\end{array}$ & $\begin{array}{l}\text { Suspicion-less } \\
\text { random drug } \\
\text { testing } \\
\text { implemented } \\
\text { in } 205 \text { school } \\
\text { districts }\end{array}$ & $\begin{array}{l}\text { School districts' responses to } \\
\text { students' first positive } \\
\text { drug test }\end{array}$ & $\begin{array}{l}\text { Appropriate responses: } \\
\text { - Refer students and parents } \\
\text { to meet with school } \\
\text { personnel and counselor } \\
\text { - Require students to receive } \\
\text { drug education and treatment }\end{array}$ \\
\hline
\end{tabular}


TABLE 3

A Summary of Findings that Oppose School Drug Testing

\begin{tabular}{|c|c|c|c|c|c|c|}
\hline Study & Study Design & Setting & Sample & Intervention & Outcomes assessed & Findings \\
\hline $\begin{array}{l}\text { Coombs and Ryan } \\
\text { (1990) }\end{array}$ & $\begin{array}{l}\text { Cross-sectional survey } \\
\text { and in-depth } \\
\text { interview }\end{array}$ & $\begin{array}{l}21 \text { intercollegiate teams, } \\
\text { UCLA, USA }\end{array}$ & $\begin{array}{l}624 \text { intercollegiate } \\
\text { athletes }\end{array}$ & $\begin{array}{c}\text { Drug testing } \\
\text { program }\end{array}$ & $\begin{array}{ll}\text { - Identification of } \\
\text { students' drug use } \\
\text { - Students' reported } \\
\text { continued drug use }\end{array}$ & $\begin{array}{l}\text { - Elevated level of drug use } \\
\text { reported in some students } \\
\text { Ways to avoid detection of } \\
\text { drugs reported in some } \\
\text { students }\end{array}$ \\
\hline $\begin{array}{l}\text { Coombs and Coombs } \\
\text { (1991) }\end{array}$ & $\begin{array}{l}\text { Cross-sectional survey } \\
\text { and in-depth } \\
\text { interview }\end{array}$ & $\begin{array}{l}21 \text { intercollegiate teams, } \\
\text { UCLA, USA }\end{array}$ & 500 student athletes & $\begin{array}{l}\text { Mandatory drug } \\
\text { testing for } \\
\text { intercollegiate } \\
\text { athletes }\end{array}$ & $\begin{array}{l}\text { - Students' morale and } \\
\text { psychological well- } \\
\text { being } \\
\text { Improvement in drug } \\
\text { testing experience }\end{array}$ & $\begin{array}{l}\text { Negative feelings reported: } \\
\text { - Embarrassed and } \\
\text { humiliated } \\
\text { Suggested improvements: } \\
\text { - } \quad \text { Orientation and education } \\
\text { : } \quad \text { Comfortable testing setting } \\
\text { - } \quad \text { Reasonable objectives } \\
\text { Rigorous testing standards }\end{array}$ \\
\hline McKinney (2002) & Cross-sectional survey & $\begin{array}{l}83 \text { high schools, Indiana, } \\
\text { USA }\end{array}$ & $\begin{array}{l}83 \text { high school } \\
\text { principals }\end{array}$ & $\begin{array}{l}\text { Mandatory, drug } \\
\text { testing in 1999- } \\
2000\end{array}$ & $\begin{array}{l}\text { Students' reported illicit drug } \\
\text { use in 2000-2001 }\end{array}$ & $\begin{array}{l}\text { Students' reported drug use } \\
\text { unchanged }\end{array}$ \\
\hline McKinney (2003) & $\begin{array}{l}\text { Cross-sectional survey } \\
\text { McKinney's (2002) } \\
\quad \text { follow-up }\end{array}$ & $\begin{array}{l}59 \text { high schools, Indiana, } \\
\text { USA }\end{array}$ & $\begin{array}{l}59 \text { high school } \\
\text { principals }\end{array}$ & $\begin{array}{l}\text { Mandatory, random } \\
\text { drug testing } \\
\text { program }\end{array}$ & $\begin{array}{l}\text { Students' reported drug use } \\
\text { when drug testing } \\
\text { reimplemented }\end{array}$ & $\begin{array}{l}\text { Reported drug use unchanged in } \\
\text { some students }\end{array}$ \\
\hline $\begin{array}{l}\text { Goldberg, Elliot, } \\
\text { MacKinnon, Moe, } \\
\text { Kuehl, Nohre, and } \\
\text { Lockwood (2003) }\end{array}$ & $\begin{array}{l}\text { Longitudinal survey } \\
\quad 1999-2000\end{array}$ & $\begin{array}{l}\text { Two high schools, } \\
\text { Oregon, USA }\end{array}$ & $\begin{array}{l}\text { Athletes vs. } \\
\text { nonathletes: } \\
\text { T1: } 276 \text { vs. } 507 \\
\text { T2: } 159 \text { vs. } 338\end{array}$ & $\begin{array}{l}\text { Mandatory drug } \\
\text { testing program } \\
\text { for student } \\
\text { athletes }\end{array}$ & $\begin{array}{l}\text { - New drug use } \\
\text { - Students' attitude and } \\
\text { beliefs on drug testing } \\
\text { - Students' attitude } \\
\text { toward school }\end{array}$ & $\begin{array}{l}\text { - No difference in new drug } \\
\text { use between control and } \\
\text { intervention schools } \\
\text { - Beliefs in reduced risk of } \\
\text { drugs increased } \\
\text { - Negative attitude toward } \\
\text { school increased }\end{array}$ \\
\hline $\begin{array}{l}\text { Yamaguchi, Johnston, } \\
\text { and O'Malley } \\
\text { (2003). }\end{array}$ & $\begin{array}{l}\text { Cross-sectional national } \\
\text { survey from } 1998 \text { to } \\
2001\end{array}$ & $\begin{array}{l}\text { Monitoring the Future } \\
\text { study } \\
\text { Youth, Education, and } \\
\text { Society study } \\
\text { USA }\end{array}$ & $\begin{array}{l}\text { Monitoring the Future } \\
\text { study: } \\
76,000 \text { students from } \\
8^{\text {th }}, 10^{\text {th }}, \& 12^{\text {th }} \\
\text { grades }\end{array}$ & $\begin{array}{l}\text { School drug testing } \\
\text { program }\end{array}$ & $\begin{array}{ll}\text { - } & \text { Prevalence of students' } \\
\text { reported illicit drug use } \\
\text { - } \quad \text { Rate of students' } \\
\text { reported marijuana use }\end{array}$ & $\begin{array}{l}\text { Drug testing not associated: } \\
\text { - } \quad \text { Prevalence of students' } \\
\text { reported illicit drug use } \\
\text { - Rate of drug use in } \\
\quad \text { experienced marijuana } \\
\quad \text { users }\end{array}$ \\
\hline $\begin{array}{l}\text { Evans, Reader, Liss, } \\
\text { Wiens, and Roy } \\
\text { (2006) }\end{array}$ & $\begin{array}{l}\text { Cross-sectional survey } \\
\text { conducted before } \\
\text { drug testing } \\
\text { implementation }\end{array}$ & $\begin{array}{l}\text { Two rural high schools, } \\
\text { North Florida, USA }\end{array}$ & $\begin{array}{l}1,011 \text { students from } \\
9^{\text {th }} \text { to } 11^{\text {th }} \text { grade }\end{array}$ & $\begin{array}{l}\text { Random suspicion- } \\
\text { less drug testing } \\
\text { program }\end{array}$ & $\begin{array}{l}\text { Students' perceived fairness } \\
\text { of drug testing policy }\end{array}$ & $\begin{array}{l}\text { Better acceptance of drug } \\
\text { testing should address: } \\
\text { - Students' perceptions of } \\
\text { peer drug use } \\
\text { D } \quad \text { Drug testing accuracy } \\
\text { Equitability of drug testing } \\
\text { consequences }\end{array}$ \\
\hline $\begin{array}{l}\text { Goldberg, Elliot, } \\
\text { MacKinnon, Moe, } \\
\text { Kuehl, Yoon, } \\
\text { Taylor, and } \\
\text { Williams (2007) }\end{array}$ & $\begin{array}{l}\text { 2-year prospective } \\
\text { randomized } \\
\text { controlled study of a } \\
\text { single cohort }\end{array}$ & $\begin{array}{l}14 \text { school districts, } \\
\text { Oregon, USA }\end{array}$ & $\begin{array}{l}653 \text { students in five } \\
\text { high schools with } \\
\text { drug testing } \\
743 \text { students in six } \\
\text { control schools }\end{array}$ & $\begin{array}{l}\text { Random drug and } \\
\text { alcohol testing in } \\
\text { high school } \\
\text { athletes }\end{array}$ & $\begin{array}{l}\text { Students' past-month } \\
\text { reported drug use }\end{array}$ & $\begin{array}{l}\text { - No deterrent effects for } \\
\text { past-month drug use in any } \\
\text { of the four follow-ups } \\
\text { - Increased risk factors for } \\
\text { future drug use }\end{array}$ \\
\hline Barrington (2008) & $\begin{array}{l}\text { Quasi-experimental, } \\
\text { mixed-methods } \\
\text { sequential } \\
\text { explanatory design }\end{array}$ & $\begin{array}{l}\text { Two rural, low-income } \\
\text { public secondary } \\
\text { school districts, USA }\end{array}$ & $\begin{array}{l}1,048 \text { high school } \\
\text { students from } 6^{\text {th }} \\
\text { to } 12^{\text {th }} \text { grade, and } \\
\text { four school } \\
\text { administrators }\end{array}$ & $\begin{array}{l}\text { Voluntary, } \\
\text { randomized, } \\
\text { student drug } \\
\text { testing program }\end{array}$ & Students' reported drug use & $\begin{array}{l}\text { Quantitative finding: } \\
\text { No significant impact on } \\
\text { students' reported drug use }\end{array}$ \\
\hline $\begin{array}{l}\text { What Works } \\
\text { Clearinghouse } \\
\text { (2008, May) }\end{array}$ & $\begin{array}{l}\text { Review of Goldberg et } \\
\text { al.'s (2007) study }\end{array}$ & $\begin{array}{c}14 \text { school districts, } \\
\text { Oregon, USA }\end{array}$ & $\begin{array}{l}653 \text { students in five } \\
\text { schools with drug } \\
\text { testing } \\
743 \text { students in six } \\
\text { control schools }\end{array}$ & $\begin{array}{l}\text { Random drug and } \\
\text { alcohol testing in } \\
\text { high sschool } \\
\text { athletes }\end{array}$ & $\begin{array}{l}\text { - Sample attrition rate } \\
\text { Demographic data of } \\
\text { sample }\end{array}$ & $\begin{array}{l}\text { Inconclusive results of Goldberg } \\
\text { et al.'s (2007) study: } \\
\text { - High attrition rate } \\
\text { - Biased sampling }\end{array}$ \\
\hline $\begin{array}{l}\text { Ringwalt, Vincus, } \\
\text { Ennett, Hanley, } \\
\text { Bowling, } \\
\text { Yacoubian, and } \\
\text { Rohrbach (2009) }\end{array}$ & $\begin{array}{l}\text { Cross-sectional survey } \\
\text { in spring } 2005\end{array}$ & $\begin{array}{l}\text { School districts from a } \\
\text { national random } \\
\text { sample, USA }\end{array}$ & $\begin{array}{l}1,612 \text { drug } \\
\text { prevention } \\
\text { coordinators from } \\
1,922 \text { school } \\
\text { districts }\end{array}$ & $\begin{array}{l}\text { Random drug } \\
\text { testing in } 205 \\
\text { school districts }\end{array}$ & $\begin{array}{l}\text { School districts' responses to } \\
\text { students' first positive } \\
\text { drug test }\end{array}$ & $\begin{array}{l}\text { Less appropriate responses: } \\
\text { - Inform law enforcement } \\
\text { - Suspension from athletic } \\
\quad \text { team or school }\end{array}$ \\
\hline
\end{tabular}

experimental schools may step up antidrug measures in schools, which would eventually exaggerate the treatment effect of school drug testing. Fourth, political and community responses to a drug testing scheme may influence student attitudes before, during, and after the implementation process. Fifth, the choice of outcome measures and honest disclosure of substance abuse behavior will definitely affect the evaluation outcomes. Sixth, as adolescent substance abuse may have a low base rate in places like Hong Kong, it may be difficult to detect real differences between the experimental group and control group unless very large sample sizes and sensitive measures are used. Finally, researchers have to consider carefully whether "blinding" can be feasibly and meaningfully carried out in related experimental studies.

The second line of research is qualitative evaluation studies. Besides those qualitative findings reported in academic journals (Tables 2 and 3), there are numerous qualitative accounts of the value and problems of school drug testing. For example, while a high school principal pointed out that "the committee worked very hard to provide a tool which would have a positive effect on our students. The extremely low number of positive tests indicates the program is worth the cost" $[10, p .1]$, Knight and Levy[11] warned that the view that drug testing in schools can prevent adolescent substance abuse has to be interpreted with caution because their efficacy has not yet been proven and drug tests are associated with significant technical concerns. 
TABLE 4

A Summary of the Quality of Studies Under Review

\begin{tabular}{|c|c|c|}
\hline Study & Study Design & Comments on the Study \\
\hline $\begin{array}{l}\text { Coombs and } \\
\text { Ryan (1990) }\end{array}$ & $\begin{array}{l}\text { Cross-sectional } \\
\text { survey and in- } \\
\text { depth interview }\end{array}$ & $\begin{array}{l}\text { - } \quad \text { Cause-effect inference could not be drawn from the findings } \\
\text { - } \quad \text { Details of group comparison not clear } \\
\text { - } \quad \text { Psychos not randomly drawn } \\
\text { - } \quad \text { Qualitative orientation of the study not clear } \\
\text { - } \quad \text { Unclear about how ideological preoccupation and biases were dealt with } \\
\text { - } \quad \text { Lualitative analysis procedures unclear } \\
\text { - }\end{array}$ \\
\hline $\begin{array}{l}\text { Coombs and } \\
\text { Coombs (1991) }\end{array}$ & $\begin{array}{l}\text { Cross-sectional } \\
\text { survey and in- } \\
\text { depth interview }\end{array}$ & $\begin{array}{l}\text { - } \quad \text { Cause-effect inference could not be drawn from the findings } \\
\text { - } \quad \text { Psychometric properties of the assessment tools unclear } \\
\text { - } \quad \text { Samples not randomly drawn } \\
\text { - } \quad \text { Qualitative orientation of the study not clear } \\
\text { - } \quad \text { Unalitative analysis procedures unclear } \\
\text { - } \quad \text { Limitations of the study not properly addressed }\end{array}$ \\
\hline $\begin{array}{l}\text { DuPont, } \\
\text { Campbell, and } \\
\text { Mazza (2002) }\end{array}$ & $\begin{array}{l}\text { Cross-sectional } \\
\text { survey } \\
\text { (quantitative } \\
\text { and qualitative } \\
\text { data collected) }\end{array}$ & $\begin{array}{l}\text { - } \quad \text { Cause-effect inference could not be drawn from the findings } \\
\text { - } \quad \text { Psychometric properties of the assessment tools unclear } \\
\text { - } \quad \text { Samples not randomly drawn } \\
\text { - } \quad \text { Qualitative orientation of the study not clear } \\
\text { - } \quad \text { Unclear about how ideological preoccupation and biases were dealt with } \\
\text { - } \quad \text { Qualitative data analysis procedures not clear } \\
\text { - } \quad \text { Limitations of the study not properly addressed }\end{array}$ \\
\hline McKinney (2002) & $\begin{array}{l}\text { Cross-sectional } \\
\text { survey }\end{array}$ & $\begin{array}{l}\text { - } \quad \text { Cause-effect inference could not be drawn from the findings } \\
\text { - } \quad \text { Psychometric properties of the assessment tools unclear } \\
\text { - } \quad \text { Samples not randomly drawn } \\
\text { - } \quad \text { Rescriptive statistics the main form of analyses } \\
\text { - } \quad \text { Findings on the impact of random student drug-testing programs not robust - } \\
\text { - } \quad \text { Limitations of the study not properly addressed }\end{array}$ \\
\hline McKinney (2003) & $\begin{array}{l}\text { Cross-sectional } \\
\text { survey } \\
\text { McKinney's } \\
\text { (2002) follow-up }\end{array}$ & $\begin{array}{l}\text { - } \quad \text { Cause-effect inference could not be drawn from the findings } \\
\text { - } \quad \text { Psychometric properties of the assessment tools unclear } \\
\text { - } \quad \text { Procedures for data collection not clear } \\
\text { - } \quad \text { Descriptive statistics the main form of analyses } \\
\text { - } \quad \text { Research report very brief } \\
\text { - } \quad \text { Alternative explanations not properly evaluated } \\
\text { - } \quad \text { Limitations of the study not properly addressed }\end{array}$ \\
\hline McKinney (2005) & $\begin{array}{l}\text { Cross-sectional } \\
\text { survey } \\
\text { McKinney's } \\
\text { (2003) follow-up }\end{array}$ & $\begin{array}{l}\text { - } \quad \text { Cause-effect inference could not be drawn from the findings } \\
\text { - } \quad \text { Psychometric properties of the assessment tools unclear } \\
\text { - } \quad \text { Hypotheses of the study not clearly stated } \\
\text { - } \quad \text { Procedures not systematically presented } \\
\text { - } \quad \text { Nescriptive statistics the main form of analyses } \\
\text { - } \quad \text { Research report very brief } \\
\text { - } \quad \text { Alternative explanations of the findings not discussed } \\
\text { - } \quad \text { Timitations of the study not properly addressed } \\
\quad \text { by the evidence presented }\end{array}$ \\
\hline Mason (2003) & $\begin{array}{l}\text { Cross-sectional } \\
\text { survey }\end{array}$ & $\begin{array}{l}\text { - } \quad \text { Cause-effect inference could not be drawn from the findings } \\
\text { - } \quad \text { Psychometric properties of the assessment tools unclear } \\
\text { - } \quad \text { Samples not randomly drawn } \\
\text { - Sample errors associated with the percentage data not properly addressed }\end{array}$ \\
\hline
\end{tabular}




\section{TABLE 4 (continued)}

\begin{tabular}{|c|c|c|}
\hline Study & Study Design & Comments on the Study \\
\hline $\begin{array}{l}\text { Goldberg, Elliot, } \\
\text { MacKinnon, } \\
\text { Moe, Kuehl, } \\
\text { Nohre, and } \\
\text { Lockwood } \\
\text { (2003) }\end{array}$ & $\begin{array}{l}\text { Longitudinal } \\
\text { survey 1999- } \\
2000\end{array}$ & $\begin{array}{l}\text { - } \quad \text { Longitudinal design commendable } \\
\text { - } \quad \text { Inclusion of a comparison school methodologically superior } \\
\text { - } \quad \text { Cause-effect inference could not be drawn from the findings } \\
\text { - } \quad \text { Some of the measures had low internal consistency } \\
\text { - } \quad \text { Samples not randomly drawn } \\
\text { - Only one experimental school and one control school involved } \\
\text { - Only mandatory drug testing among the athletes focused upon } \\
\text { - } \quad \text { Only quantitative data collected } \\
\text { - Subject attrition effect not fully explored }\end{array}$ \\
\hline $\begin{array}{l}\text { Evans, Reader, } \\
\text { Liss, Wiens, and } \\
\text { Roy (2006) }\end{array}$ & $\begin{array}{l}\text { Cross-sectional } \\
\text { survey } \\
\text { conducted } \\
\text { before drug } \\
\text { testing } \\
\text { implementation }\end{array}$ & $\begin{array}{l}\text { - } \quad \text { Cause-effect inference could not be drawn from the findings } \\
\text { - } \quad \text { Reliability of the } 10 \text {-item measure not particularly high } \\
\text { - } \quad \text { Salidity of the } 10 \text {-item measure not clear } \\
\text { - } \quad \text { Predictors of fairness attitude and policy effectiveness belief examined } \\
\text { - } \quad \text { Limitations of the study discussed } \\
\text { - } \quad \text { Only quantitative data collected }\end{array}$ \\
\hline $\begin{array}{l}\text { Evans-Whipp, } \\
\text { Bond, } \\
\text { Toumbourou, } \\
\text { and Catalano } \\
\text { (2007) }\end{array}$ & $\begin{array}{l}\text { Cross-sectional } \\
\text { survey }\end{array}$ & $\begin{array}{l}\text { - } \quad \text { Cause-effect inference could not be drawn from the findings } \\
\text { - } \quad \text { Students, parents, and administrators recruited } \\
\text { - } \quad \text { Rarge sample size in different samples } \\
\text { - } \quad \text { Psychom and representative samples drawn } \\
\text { - } \quad \text { Both descriptive and inferential statistical analyses conducted } \\
\text { - } \quad \text { Limitations of the study discussed } \\
\text { - Only quantitative data collected }\end{array}$ \\
\hline $\begin{array}{l}\text { Goldberg, Elliot, } \\
\text { MacKinnon, } \\
\text { Moe, Kuehl, } \\
\text { Yoon, Taylor, } \\
\text { and Williams } \\
\text { (2007) }\end{array}$ & $\begin{array}{l}\text { Two-year } \\
\text { prospective } \\
\text { randomized } \\
\text { controlled study } \\
\text { of a single } \\
\text { cohort }\end{array}$ & $\begin{array}{l}\text { - } \quad \text { Two-year prospective randomized controlled study } \\
\text { - } \quad \text { Reliability of measures acceptable } \\
\text { - } \quad \text { Limititations of the study discussed } \\
\text { - } \quad \text { Linear mixed models not employed } \\
\text { - } \quad \text { Only quantitative data collected }\end{array}$ \\
\hline Barrington (2008) & $\begin{array}{l}\text { Quasi- } \\
\text { experimental } \\
\text { mixed-methods } \\
\text { sequential } \\
\text { explanatory } \\
\text { design }\end{array}$ & $\begin{array}{l}\text { - } \quad \text { A mixed-method design adopted } \\
\text { - } \quad \text { Salidated measures used } \\
\text { - } \quad \text { Qualitative orientation of the study not clear } \\
\text { - Unclear about how ideological preoccupation and biases were dealt with } \\
\text { - } \quad \text { Limitations of the study addressed }\end{array}$ \\
\hline $\begin{array}{l}\text { Ringwalt, Vincus, } \\
\text { Ennett, Hanley, } \\
\text { Bowling, } \\
\text { Yacoubian, and } \\
\text { Rohrbach } \\
\text { (2009) }\end{array}$ & $\begin{array}{l}\text { Cross-sectional } \\
\text { survey in spring } \\
2005\end{array}$ & $\begin{array}{l}\text { - } \quad \text { Cause-effect inference could not be drawn from the findings } \\
\text { - } \quad \text { Random samples selected } \\
\text { - } \quad \text { Descriptive statistics the main form of analyses } \\
\text { - } \quad \text { Design and statistical analyses strong } \\
\text { - } \quad \text { Limitations of the study addressed } \\
\text { - Only quantitative data collected }\end{array}$ \\
\hline $\begin{array}{l}\text { Yamaguchi, } \\
\text { Johnston, and } \\
\text { O'Malley (2003). }\end{array}$ & $\begin{array}{l}\text { Cross-sectional } \\
\text { national survey } \\
\text { from } 1998 \text { to } \\
2001\end{array}$ & $\begin{array}{l}\text { - } \quad \text { Cause-effect inference could not be drawn from the findings } \\
\text { - } \quad \text { Large sample size in different samples } \\
\text { - } \quad \text { Psierarchical linear models examined } \\
\text { - } \quad \text { Background confounding factors not properly examined } \\
\text { - } \quad \text { Limitations of the study discussed } \\
\text { - } \quad \text { Only quantitative data collected }\end{array}$ \\
\hline $\begin{array}{l}\text { What Works } \\
\text { Clearinghouse } \\
\text { (2008, May) }\end{array}$ & $\begin{array}{l}\text { Review of } \\
\text { Goldberg et al.'s } \\
\text { (2007) study }\end{array}$ & $\begin{array}{l}\text { - } \quad \text { Sample attrition problem leading to bias } \\
\text { - } \quad \text { Initial differences questionnaires leading to bias } \\
\text { confounding effect } \\
\text { - The conclusion of the study does not conform to What Works Clearinghouse } \\
\text { standards }\end{array}$ \\
\hline
\end{tabular}


When researchers conduct qualitative evaluations of school drug testing, it is important to pay particular attention to the rigor of the studies. Shek et al.[12] pointed out that there are 12 principles that should be upheld in qualitative evaluation studies: (1) statement of the philosophical base of the study; (2) justification for the number and nature of the participants of the study; (3) detailed description of data collection procedures; (4) discussion of biases in the study; (5) description of steps taken to guard against biases or arguments that biases should and/or could not be eliminated; (6) pay attention to reliability issues; (7) considering triangulation strategies; (8) use of peer checking and member checking; (9) use of audit trails; (10) examination of alternative explanations; (11) accounting for negative evidence; and (12) examination of limitations of the study. Obviously, methodological rigor of future qualitative evaluation studies in this field can be strengthened by upholding these principles.

Adopting a balanced perspective, school drug testing schemes may not be a panacea for adolescent substance abuse. In the long run, effort should be made to integrate school drug testing with other preventive measures, such as preventive drug education and positive youth development[13,14,15,16,17], to help young people to stay away from drugs. Fundamentally, it is important to take an evidence-based approach to evaluate the strategies to tackle adolescent substance abuse, including a school drug testing scheme.

\section{ACKNOWLEDGMENTS}

The preparation for this paper was financially supported by The Hong Kong Jockey Club Charities Trust.

\section{REFERENCES}

1. Shek, D.T.L. (2007) Tackling adolescent substance abuse in Hong Kong: where we should go and should not go? TheScientificWorldJOURNAL 7, 2021-2030.

2. Shek, D.T.L., Ed. (2006) International Conference on Tackling Drug Abuse: Conference Proceedings. Narcotics Division, Government of the Hong Kong Special Administrative Region, P.R.C.

3. Johnston, L.D., O'Malley, P.M., Bachman, J.G., and Schulenberg, J.E. (2009) Monitoring the Future National Survey Results on Drug Use, 1975-2008. Volume I: Secondary School Students. NIH Publication No. 09-7402. National Institute on Drug Abuse, Bethesda, MD.

4. Office of Applied Studies, Substance Abuse and Mental Health Services Administration, U.S. Department of Health and Human Services. Results from the 2008 National Survey on Drug Use and Health: National Findings. Available from http://www.oas.samhsa.gov/nsduh/2k8nsduh/2k8Results.cfm\#2.2

5. Office of National Drug Control Policy (2004) What You Need to Know about Starting a Student Drug-Testing Program. Drug Policy Information Clearing House, Washington, DC. pp. v-vi, 18-19.

6. Roche, A.M., Bywood, P., Pidd, K., Freeman, T., and Steenson, T. (2009) Drug testing in Australian schools: policy implications and considerations of punitive, deterrence and/or prevention measures. Int. J. Drug Policy 20, 521-528.

7. Goldberg, L., Elliot, D.L., MacKinnon, D.P., Moe, E.L., Kuehl, K.S., Yoon, M., Taylor, A., and Williams, J. (2007) Outcomes of a prospective trial of student-athlete drug testing: the student athlete testing using random notification (SATURN) study. J. Adolesc. Health 41, 421-429.

8. $\quad$ Knight, J.R. and Levy, S. (2007) Editorial: the national debate on drug testing in schools. J. Adolesc. Health 41, 419420.

9. Yamaguchi, R., Johnston, L.D., and O'Malley, P.M. (2003) Relationship between student illicit drug use and school drug-testing policies. J. Sch. Health 73(4), 159-164.

10. Nighswonger, G. (2007) Random Drug Testing. Scottsbluff High School. Bluffs Middle School. $2006-2007$. Available from http://www.sbps.net/pages/uploaded_files/9_a_RandomDrugTestingSummary2007_0.pdf

11. Knight, J. R. and Levy, S. (2005) An F for School Drug Tests. Boston Globe. Available from http://www.boston.com/news/globe/editorial_opinion/oped/articles/2005/06/13/an_f_for_school_drug_tests/

12. Shek, D.T.L., Tang, V., and Han, X.Y. (2005) Quality of qualitative evaluation studies in the social work literature: evidence that constitutes a wakeup call. Res. Soc. Work Pract. 15, 180-194.

13. Shek, D.T.L. and Ng, C.S.M. (2009) Subjective outcome evaluation of the Project P.A.T.H.S. (Secondary 2 Program): views of the program participants. TheScientificWorldJOURNAL 9, 1012-1022.

14. Shek, D.T.L., Sun, R.C.F., and Tang, C.Y.P. (2009) Experimental implementation of the Secondary 3 Program of Project P.A.T.H.S.: observations based on the co-walker scheme. TheScientificWorldJOURNAL 9, 1003-1011. 
15. Shek, D.T.L., Ma, H.K., and Sun, R.C.F. (2008) Interim evaluation of the Tier 1 Program (Secondary 1 Curriculum) of the Project P.A.T.H.S.: first year of the Full Implementation Phase. TheScientificWorldJOURNAL 8, 47-60.

16. Shek, D.T.L., Ed. (2008) Special issue: evaluation of Project P.A.T.H.S. in Hong Kong. TheScientificWorldJOURNAL 8, 1-94.

17. Shek, D.T.L. and Ma, H.K. (2010) Editorial: evaluation of the Project P.A.T.H.S. in Hong Kong: are the findings replicable across different populations? TheScientificWorldJOURNAL: TSW Child Health \& Human Development 10, 178-181.

This article should be cited as follows:

Shek, D.T.L. (2010) School drug testing: a critical review of the literature. TheScientificWorldJOURNAL: TSW Child Health \& Human Development 10, 356-365. DOI 10.1100/tsw.2010.31. 


\section{APPENDIX 1}

\section{Studies and Articles Under Review}

\begin{tabular}{|c|c|c|}
\hline Review Authors & Year & Publication Details \\
\hline $\begin{array}{l}\text { Ringwalt, C., Vincus, A.A., } \\
\text { Ennett, S.T., Hanley, S., } \\
\text { Bowling, J.M., } \\
\text { Yacoubian, G.S., and } \\
\text { Rohrbach, L.A. }\end{array}$ & 2009 & $\begin{array}{l}\text { Responses to positive results from suspicion-less random drug tests in } \\
\text { US public school districts. J. Sch. Health } 79(4), 177-183 \text {. }\end{array}$ \\
\hline Barrington, $\mathrm{K}$. & 2008 & $\begin{array}{l}\text { Voluntary, randomized, student drug-testing: impact in a rural, low- } \\
\text { income, community. J. Alcohol Drug Educ. 52(1), 47-66. }\end{array}$ \\
\hline $\begin{array}{l}\text { What Works } \\
\text { Clearinghouse }\end{array}$ & $\begin{array}{l}\text { 2008, } \\
\text { May }\end{array}$ & $\begin{array}{l}\text { WWC quick review of the article "Outcomes of a Prospective Trial of } \\
\text { Student-Athlete Drug Testing: The Student Athlete Testing Using } \\
\text { Random Notification (SATURN) Study". Institute of Education Science: } \\
\text { U.S. Department of Education. Retrieved from } \\
\text { http://www.ies.ed.gov/ncee/wwc/publications/quickreviews/Satu } \\
\text { rn/index.asp }\end{array}$ \\
\hline $\begin{array}{l}\text { Evans-Whipp, T.J., Bond, } \\
\text { L., Toumbourou, J.W., } \\
\text { and Catalano, R.F. }\end{array}$ & 2007 & $\begin{array}{l}\text { School, parent, and student perspectives of school drug policies. J. Sch. } \\
\text { Health } 77(3), 138-146,153-154 \text {. }\end{array}$ \\
\hline $\begin{array}{l}\text { Goldberg, L., Elliot, D. L., } \\
\text { MacKinnon, D.P., Moe, } \\
\text { E., Kuehl, K.S., Nohre, } \\
\text { L., and Lockwood, C.M. }\end{array}$ & 2007 & $\begin{array}{l}\text { Outcomes of a prospective trial of student-athlete drug testing: the } \\
\text { Student Athlete Testing Using Random Notification (SATURN) study. } \\
\text { J. Adolesc. Health 41, 421-429. }\end{array}$ \\
\hline $\begin{array}{l}\text { Evans, G.D., Reader, S., } \\
\text { Liss, H.J., Wiens, B.A., } \\
\text { and Roy, A. }\end{array}$ & 2006 & $\begin{array}{l}\text { Implementation of an aggressive random drug-testing policy in a rural } \\
\text { school district: student attitudes regarding program fairness and } \\
\text { effectiveness. J. Sch. Health 76(9), 452-458. }\end{array}$ \\
\hline McKinney, J.R. & 2005 & $\begin{array}{l}\text { Effectiveness of Student Random Drug-Testing Programs. The Student } \\
\text { Drug-Testing Coalition. U.S. }\end{array}$ \\
\hline $\begin{array}{l}\text { Goldberg, L., Elliot, D.L., } \\
\text { MacKinnon, D.P., Moe, } \\
\text { E.L., Kuehl, K.S., Yoon, } \\
\text { M., Taylor, A., and } \\
\text { Williams, J. }\end{array}$ & 2003 & $\begin{array}{l}\text { Drug testing athletes to prevent substance abuse: background and pilot } \\
\text { study results of the SATURN study. J. Adolesc. Health 32, 16-25. }\end{array}$ \\
\hline Mason, $\mathrm{K}$. & 2003 & $\begin{array}{l}\text { Drug Testing in Schools: Attitudes of High School Students [Ph.D. } \\
\text { thesis]. University of New Orleans. (Cited in McKeganey, N. [2005] } \\
\text { Random Drug Testing of School Children: A Shot in the Arm or a Sho } \\
\text { in the Foot for Drug Prevention. Joseph Rowntree Foundation, York. } \\
\text { pp. 7-8). }\end{array}$ \\
\hline McKinney, J.R. & 2003 & $\begin{array}{l}\text { The Effectiveness of Random Drug Testing Programs: A Statewide } \\
\text { Follow-Up Study. The Student Drug-Testing Coalition. U.S. }\end{array}$ \\
\hline $\begin{array}{l}\text { Yamaguchi, R., Johnston, } \\
\text { L.D., and O'Malley, P.M. }\end{array}$ & 2003 & $\begin{array}{l}\text { Relationship between student illicit drug use and school drug-testing } \\
\text { policies. J. Sch. Health 73(4), 159-164. }\end{array}$ \\
\hline $\begin{array}{l}\text { DuPont, R.L., Campbell, } \\
\text { T.G., and Mazza, J.J. }\end{array}$ & 2002 & $\begin{array}{l}\text { Report of a Preliminary Study: Elements of a Successful School-Based } \\
\text { Student Drug Testing Program. The Institute for Behavior and Health. } \\
\text { U.S. }\end{array}$ \\
\hline McKinney, J.R. & 2002 & $\begin{array}{l}\text { The Effectiveness and Legality of Random Drug Testing Policies. The } \\
\text { Student Drug-Testing Coalition. U.S. }\end{array}$ \\
\hline $\begin{array}{l}\text { Coombs, R.H. and } \\
\text { Coombs, C.J. }\end{array}$ & 1991 & $\begin{array}{l}\text { The impact of drug testing on the morale and well-being of mandatory } \\
\text { participants. Int. J. Addict. 26(9), 981-992. }\end{array}$ \\
\hline $\begin{array}{l}\text { Coombs, R.H. and Ryan, } \\
\text { F.J. }\end{array}$ & 1990 & $\begin{array}{l}\text { Drug testing effectiveness in identifying and preventing drug use. Am. J. } \\
\text { Drug Alcohol Abuse 16, 173-184. }\end{array}$ \\
\hline
\end{tabular}

